\section{Conference calendar: is less more?}

\section{The summer conference season is now in full swing. As scientists dash from session to session and venue to venue, now is a good time to take stock of what conference attendance seeks to achieve.}

Scientific conferences aim to bring researchers together to share recent data, generate new ideas, foster collaborations, and facilitate interactions between scientists in various stages of their careers. Cell biology is rife with anecdotes about major breakthroughs growing out of discussions over a beer in a remote location. Meetings also provide a crucial forum for debating differing viewpoints and create an opportunity for the broader community to weigh in on contentious issues. They may also allow scientists to mull over their research projects without the distraction of additional academic responsibilities. With the extreme competition for tenure-track positions and funding, younger scientists are now under pressure to network and share their recent findings. Wellattended international conferences represent an ideal location for all these endeavours.

The increasingly collaborative nature of biological research, and the cross-fertilization of fields, has spawned an intensely crowded conference calendar for molecular and cell biologists. Organisations such as the Cold Spring Harbor Laboratories (CSHL) and the Gordon Research Conferences (GRC) have been running meetings for about 75 years, and have expanded their reach to European and Asian venues. The first Keystone symposia in California and advanced training programmes at the EMBL in Heidelberg, Germany were established in the 1970s, and continue to flourish. The long-running summer courses held at the CSHL and at the MBL in Woods Hole, Massachusetts, are complemented by EMBO and Wellcome Trust courses in Europe. Joining these on the conference calendar are the numerous society meetings, as well as conferences sponsored by individual institutes, academic consortia, journals and reagents companies.

Each of these meetings represents an excellent opportunity for researchers, but what is the optimal number of conferences that allow scientists to achieve a healthy balance between networking with their colleagues and communicating their new work, and meeting the demands of their weekly workload? With research budgets still tight around the world, do conferences remain a sound investment for faculty, post-docs and students? Should the current culture of packed conference travel schedules, which often means that researchers are away from the lab for weeks on end, be encouraged?
Given the bulging conference calendar, researchers who wish to attend multiple meetings each summer are often unable to attend each conference in full. Many attend only for a day or two, and even then struggle to be present at all the sessions. Conference attendees also increasingly use the talk-free sessions at meetings, which are designed to facilitate communication between attendees, as an opportunity to meet their other academic commitments. As a result, it can be difficult for researchers to find the time and occasion to interact with their colleagues. Sparse conference attendance by senior and junior researchers alike can greatly reduce the overall quality of a conference.

A hectic travel schedule can also tip the balance too far away from responsibilities in the lab. In particular, mentoring of students and post-docs (http://www.nature.com/ncb/journal/v12/n2/full/ncb0210101.html) can suffer when the PI is absent for long periods. This is likely to be more of an issue for junior PIs, who might not have experienced senior members in the lab who can hold down the fort for them. For researchers actively engaged in bench work, a week out of town precipitates a logistical challenge in managing their research project. Clearly, researchers must strike the appropriate balance between their presence at conferences and in the lab, as both are crucial for a successful and engaging scientific career - for PIs and for their students and post-docs.

Conference organizers could take certain steps to encourage attendees to stay for the entire meeting and immerse themselves in the proceedings. In research areas that are well-served by multiple meetings per year, organizations could consolidate and integrate their meetings with the rest of the landscape, or decrease the frequency of the conferences. The initiative by the GRC to hold meetings every two years is a step in this direction. Shorter meetings might also redress this balance particularly in research areas with many meetings. Meeting organizers must also strike a better balance between the need to create a schedule that allows a sufficient number of researchers to present talks or poster sessions, and conference length. A long conference can often become unproductive as it carries on; more often than not, the crowd starts getting restless by day three, as evening sessions (and post-session conversations at the bar) begin to take their toll. Organizers should continue to encourage the presentation of unpublished data, and should also consider not advertising the meeting programme ahead of time, as done at CSHL meetings. These approaches could increase the incentive for attendees to stay for the duration of the conference.

Conferences represent the major avenue for scientists to gather with their colleagues and communicate with each other. Considered decisions about not overcrowding the conference landscape with conferences that are redundant in scope and mission, and finding ways to improve attendance at existing ones may go a long way towards a more enriching conference experience. 